\title{
In situ manipulation of scanning tunneling microscope tips without tip holder
}

C. Raad, K. H. Graf, and Ph. Ebert

Citation: Review of Scientific Instruments 81, 013706 (2010);

View online: https://doi.org/10.1063/1.3284508

View Table of Contents: http://aip.scitation.org/toc/rsi/81/1

Published by the American Institute of Physics

\section{Articles you may be interested in}

Two-step controllable electrochemical etching of tungsten scanning probe microscopy tips

Review of Scientific Instruments 83, 063708 (2012); 10.1063/1.4730045

Scanning Auger microscopy study of $\mathrm{W}$ tips for scanning tunneling microscopy

Review of Scientific Instruments 74, 3368 (2003); 10.1063/1.1581392

Generation of constant-amplitude radio-frequency sweeps at a tunnel junction for spin resonance STM Review of Scientific Instruments 87, 074703 (2016); 10.1063/1.4955446

Etching of Cr tips for scanning tunneling microscopy of cleavable oxides Review of Scientific Instruments 88, 023705 (2017); 10.1063/1.4976567

Upgrade of a commercial four-probe scanning tunneling microscopy system

Review of Scientific Instruments 88, 063704 (2017); 10.1063/1.4986466

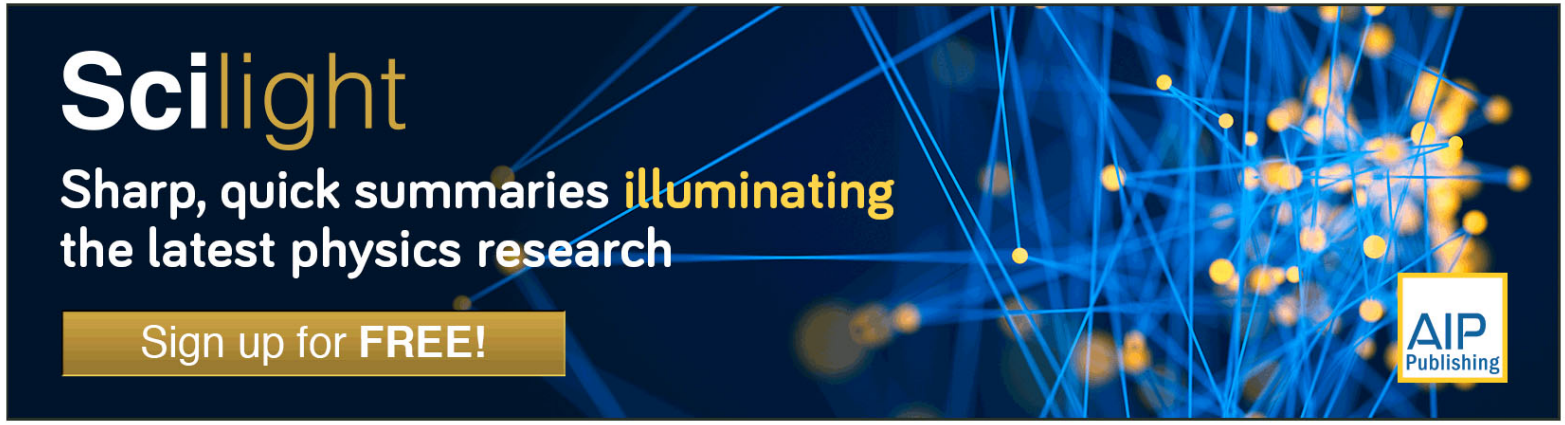




\title{
In situ manipulation of scanning tunneling microscope tips without tip holder
}

\author{
C. Raad, K. H. Graf, and Ph. Ebert ${ }^{\text {a) }}$ \\ Institut für Festkörperforschung, Forschungszentrum Jülich GmbH, 52425 Jülich, Germany
}

(Received 7 November 2009; accepted 11 December 2009; published online 25 January 2010)

\begin{abstract}
A design for a manipulator system for manipulating bare scanning tunneling microscopy (STM) tips without any tip holder is presented. The extremely stiff and rigid system consists of an ultrahigh vacuum compatible fully three-dimensionally movable gripper module driven by stepping motors and piezomotors. The tips are clamped by hardened tool steel gripper jaws, which are controlled by a stepping motor through levers. The system allows the reproducible manipulation of bare tungsten tips made of wires with diameters of $0.25 \mathrm{~nm}$ and having length of only up to $3 \mathrm{~mm}$ without damaging the tip or the STM. The tip manipulators' advantage is that the total mass of the scanning piezotube is reduced by removing the mass of a separate tip holder. Thereby, it becomes possible to further increase the resonance frequencies of the STM. (c) 2010 American Institute of Physics.

[doi:10.1063/1.3284508]
\end{abstract}

\section{INTRODUCTION}

Scanning tunneling microscopy (STM) and spectroscopy ${ }^{1}$ are an exceptional tool for the atomically resolved structural and electronic characterization of surfaces. ${ }^{2,3}$ However, STM is not only limited to unravel and understand surface reconstructions. A wide range of topics has been addressed in recent years, highlighting the exceptional status of STM as versatile atomically resolving probe. For example, the high spatial resolution combined with local spectroscopy allowed to investigate atomic sized features, such as point defects, ${ }^{4}$ bulk dopant atoms, ${ }^{5}$ or dislocations exposed close to the surface. ${ }^{6}$ In a further development, the so-called cross-sectional STM technique made nanostructures and internal interfaces in semiconductors accessible by cleavage through cross-sectional planes of the semiconductor structures. ${ }^{7}$ This technique allowed to investigate with atomic resolution the compositional distributions as well as roughness of semiconductor interfaces ${ }^{8}$ and related nanostructures, such as quantum $\operatorname{dots}^{9}$ and provides a distinction between atomic (chemical) and electronic interfaces. ${ }^{10}$ The STM is in addition sensitive to potential fluctuations and thereby allows to probe the local potential, e.g., around charged defects ${ }^{11}$ or induced by fluctuations in the dopant distribution. $^{12}$ Beyond electronic sensitivity, the spinpolarized STM, based on specially prepared magnetic tips, ${ }^{13}$ provides even an atomically resolved view of the spin structure of surfaces. ${ }^{14}$

All these applications of STM rely on the same essential ingredient: the metallic probe tip. Its quality determines almost completely the achievable spatial, energetic, or magnetic resolution and thus the quality of the scientific conclusions. Therefore, large efforts were undertaken to improve the conditions of the tips, e.g., by thermal treatments, ion sputtering, electron bombardment, or field emission ${ }^{15}$ or even prepare special magnetic tips by suitable coating techniques. ${ }^{13}$ For this, the tip needs to be moved from the

\footnotetext{
${ }^{a)}$ Electronic mail: p.ebert@fz-juelich.de.
}

STM head to the separate treatment stages within the ultrahigh vacuum system because the high voltages, the ion bombardment, or the high temperatures used to improve the condition of the tip would damage the STM itself. In order to facilitate the manipulation of the tips, the tips are typically inserted into tip holders, ${ }^{16}$ which can be manipulated more conveniently. Unfortunately, the mass of the tip holders increases the mass, which needs to be moved during scanning of the tip over the sample surface. Thereby the resonance frequency is reduced and the achievable scanning speed is lowered. Since many physically important effects on surfaces are connected to the dynamics of the surface atoms, ${ }^{17}$ a reduced scanning speed also diminishes the physical information within the STM data. Therefore, we developed a method, which allows us to manipulate bare STM tips without any tip holder. This allows us to minimize the mass of the scanning piezotube and maximize the resonance frequency.

\section{DESIGN}

First we recall the technical requirements set by the STM technique itself. Most scanning tunneling microscopes are not used in air, but rather under controlled environments, such as ultrahigh vacuum. Furthermore, due to the desired atomic resolution of the STM a highly vibration free environment is required. Therefore, the STM and the vacuum system are typically suspended separately on vibration damping systems. Thus, any tip manipulation system, which is attached to the vacuum chamber, would need to accommodate the relative motion and vibrations between the chamber and the separately damped STM. The risk of damaging the STM by uncontrolled motions between the STM and the tip manipulation can be avoided, by mounting the manipulation directly on the same ground plate on which the STM rests. This also prevents that external vibrations can couple to the STM by short circuiting the vibration damping.

The design of the tip manipulator is further geometrically restricted by the limited space provided in the vacuum 


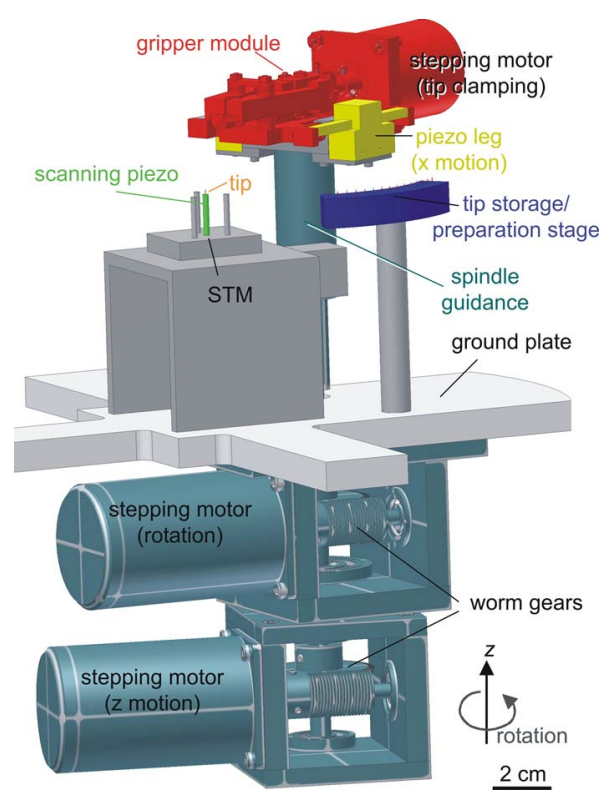

FIG. 1. (Color online) Schematic overview of the design of the tip manipulation system without use of tip holders. The manipulator is mounted on the ground plate on which the STM rests. Below the ground plate are two stepping motor-worm gear units for vertical motion ( $z$-direction) and rotation. This is realized using a spindle guidance. On top is the gripper module, with which a tip can be removed or inserted from the scanning piezo and moved to a tip storage or tip preparation site.

system. In addition, the construction needs to be exceptionally rigid, in order to avoid the introduction of vibrations from the tip manipulator itself, and perfectly aligned. Vibrations would on the one hand directly affect the scanning process. On the other hand vibration induced tilts strongly increase the risk to break the scanning piezotube because a tilted nonaligned tip exerts radial forces on the piezotube while being inserted into or removed from it. Finally, the ultrahigh vacuum environment requires also that all the components used in the tip manipulator are vacuum compatible, including stepping motors and gears.

These technical requirements lead us to develop the design illustrated in Fig. 1. We used a modified Besocke type STM (Ref. 18) for the demonstration of the tip manipulation. The STM is rigidly attached to a ground plate, which itself is mounted on a damping system within the vacuum chamber. On the ground plate the tip manipulator is rigidly attached and perpendicularly aligned. In order to approach and withdraw the tip manipulator, we need four motions: three linear and one rotation. This allows a full three-dimensional manipulation motion of the tips within the ultrahigh vacuum chamber.

Two of the four motions ( $z$ motion and rotation) are realized by a spindle guidance system (Fig. 2), which is driven by two stepping motors combined with worm gears (see bottom parts in Fig. 1). One of the stepping motor-worm gear units (upper unit) drives the rotation of the whole manipulation system. The lower unit drives the inner spindle relative to the nut of the spindle guidance system and thereby shifts the holder of the gripper module up and down. In order to avoid a cold shut under ultrahigh vacuum conditions, a stainless steel-bronze combination is used for the spindle guid-

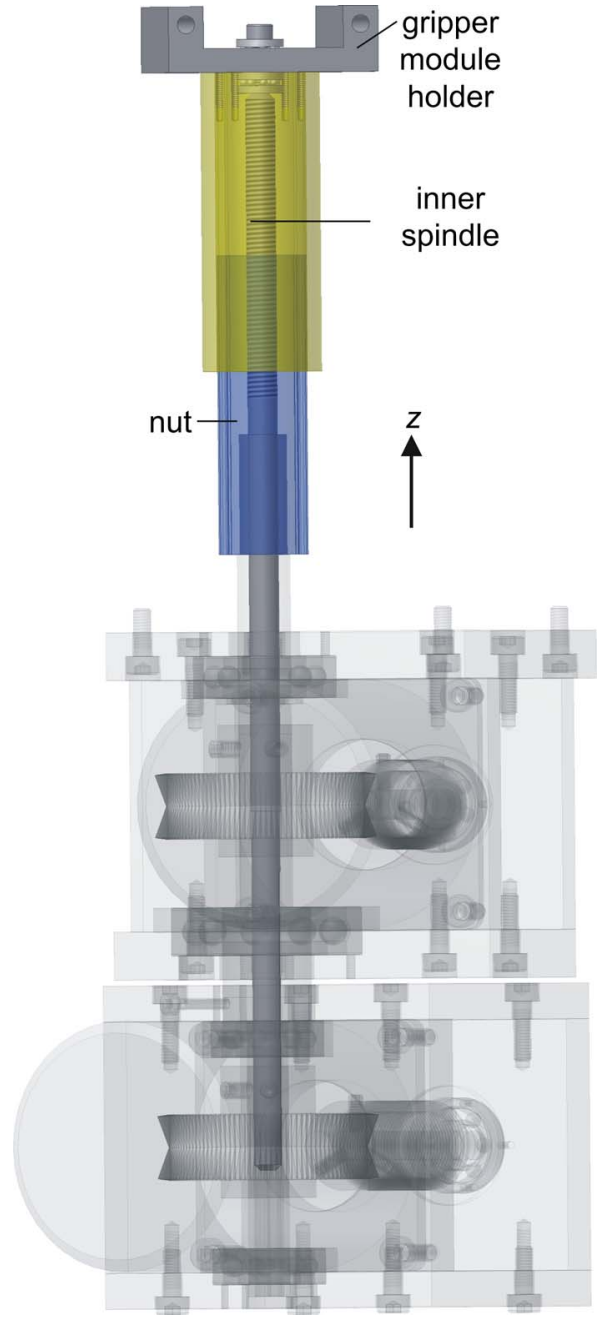

FIG. 2. (Color online) Schematic view of the spindle guidance system used for the $z$-motion and rotation of the gripper module. The gripper module holder is moving up and down, when the inner spindle turns. The whole system is rotated by rotating the nut. The nut is made of bronze, while the other parts are made of stainless steel.

ance system (nut in bronze). The spindle guidance system is extremely rigid, such that no bending or loosening can occur and potentially damage the STM.

The stepping motors need to have an extremely small stepping width, in order to manipulate the tip in extra fine steps, such that $0.25 \mathrm{~mm}$ tungsten $(\mathrm{W})$ wire tips can be inserted in approximately $0.26 \mathrm{~mm}$ holes with an accuracy of better than $0.01 \mathrm{~mm}$. Otherwise, radial forces appear and lead to a cracking of the scanning piezotube. Therefore, we chose multipolar stepping motors with approximately 40000 steps per turn. Furthermore, the worm gears need to be free from backlash. We realized this by using prestressed gear wheels in the worm gears. This yields the needed reproducibility of the tip motions for repeatedly accurate approaches to the scanning tube.

Note, the two stepping motor worm gear units are placed below the ground plate, in order to keep a maximum of space free in the STM area and reduce the space needed in the vacuum chamber. The stepping motors and all the related components of the manipulator are vacuum compatible.

The gripper module (shown schematically in Fig. 3) is 


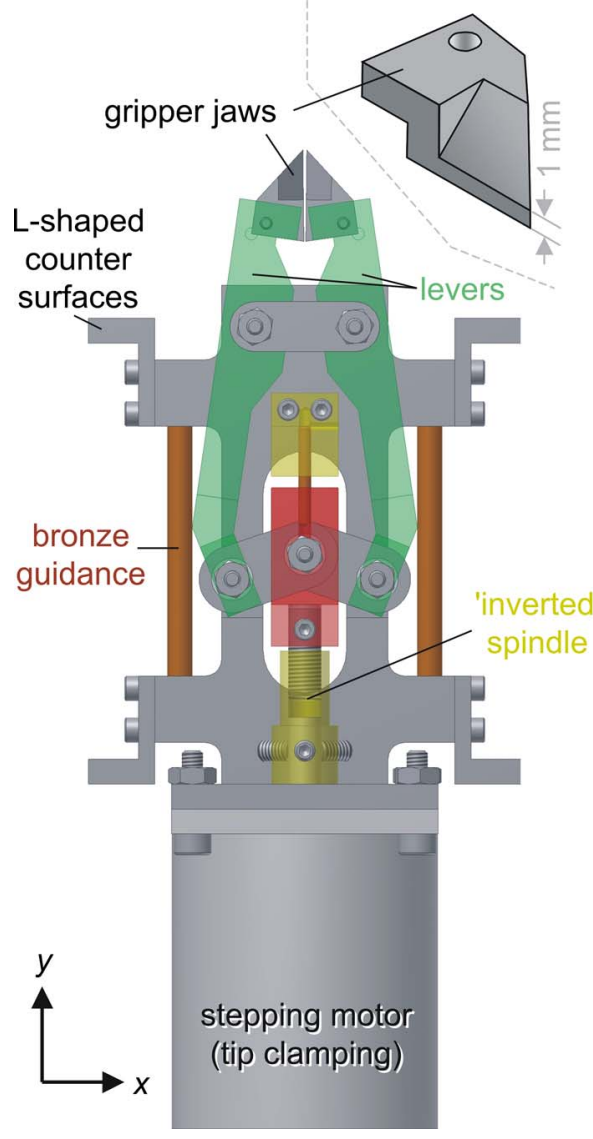

FIG. 3. (Color online) Schematic view of the gripper module. The stepping motor drives the motion of the gripper jaws (see inset for the shape of the jaws) through an inverted spindle, where the outer part (not the spindle) rotates and moves two levers. The gripper jaws are made of hardened tool steel, in order to avoid that the $\mathrm{W}$ tips damage the jaws during manipulation, because of the high clamping forces used. The gripper module is shifted forward and backward using piezolegs indicated in Fig. 1.

mounted by inserting its two bronze guidances into the stainless steel gripper module holder on top of the spindle guidance system described above. The material combination is again needed to avoid cold shuts. The gripper module is moved for- and backward in $y$ direction (along the bronze guidances) by piezomotors (piezolegs). These push against the L-shaped countersurfaces. The piezolegs have the advantage to allow accurate and reproducible small motions. The gripper module itself consists of a stepping motor driving the gripper jaws through an inverted spindle (outer part is turned) driven set of levers ( $x$ motion). At the ends of each levers a jaw is mounted (inset in Fig. 3). Since we have to manipulate $\mathrm{W}$ tips of $0.25 \mathrm{~mm}$ in diameter and with lengths of 2-3 mm, the jaws need to be delicate and simultaneously exhibit a high stiffness and a hardness comparable with that of W. Therefore, we used hardened tool steel and a special design of the jaw shape, where the clamping surface is only $1 \mathrm{~mm}$ high, but the material supporting behind rapidly gains in size to distribute the high clamp forces (see inset in Fig. 3 ). Note, the clamp forces used are rather high because one needs to ensure that the tip, once clamped in the gripper module, does not shift or tilt because the strictly needed $z$ alignment will be lost immediately. This will inevitably in-
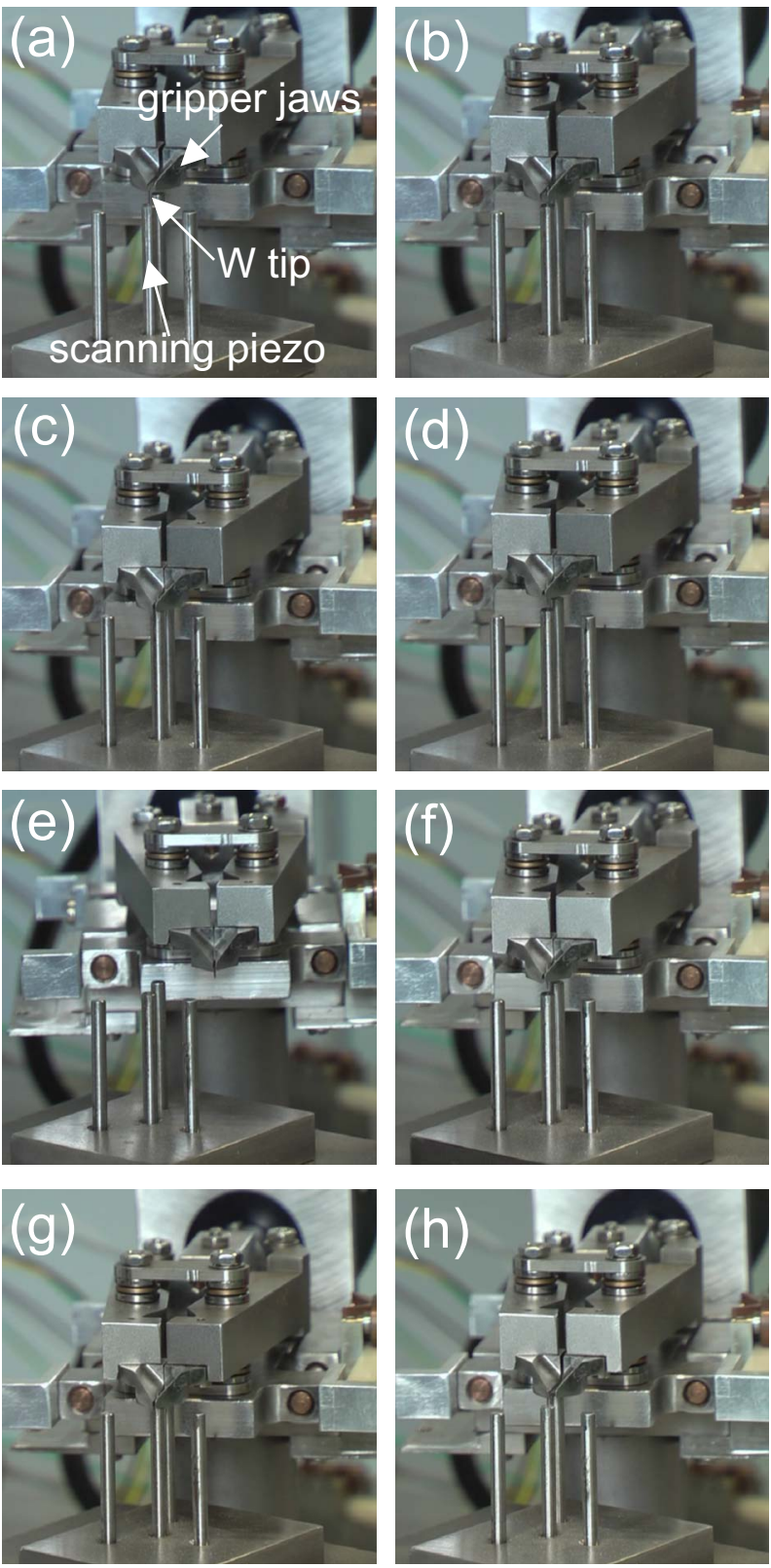

FIG. 4. (Color online) Individual frames takes out of a movie, where the tip is removed and inserted into the test scanning piezotube. [(a) and (b)] Approach of the gripper jaws toward the tip. (c) Clamping of the tip. (d) Removal of the tip by vertically lifting the gripper module. (e) Transfer of the tip to any desired place. (f) Reapproach of the tip toward the scanning piezotube. (g) Insertion of the tip. (h) Retraction of the gripper module.

duce radial forces on the scanning piezotube and thus damage the STM.

The final part of the system is a tip storage and/or modification stage, in which the desired tip preparation can be performed without endangering the STM by high voltages, temperatures of ion/electron bombardment. This storage stage can be modified easily to be transferable through a load lock into and out of the vacuum system, without need to break the vacuum.

\section{RESULTS}

Figures 4 and 5 show selected frames taken from movies of the tip manipulation process. First we focus on the details 

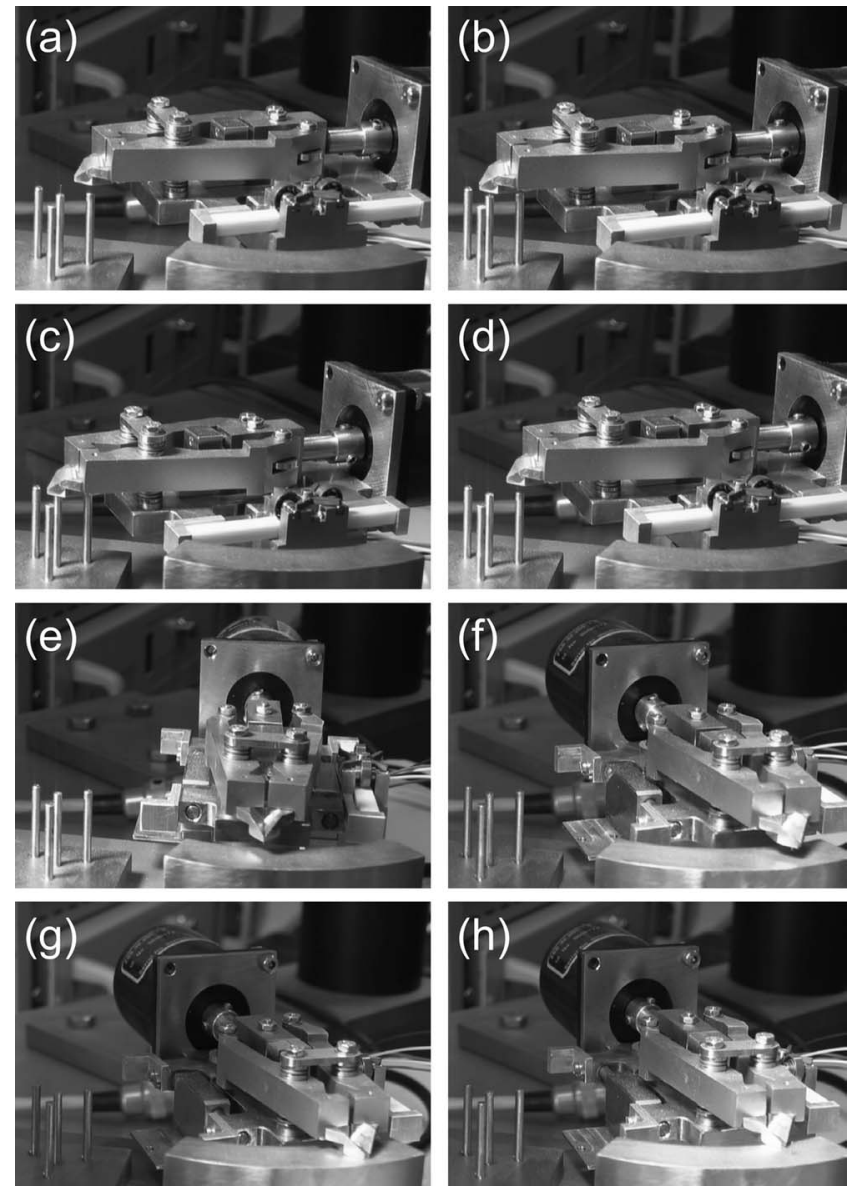

FIG. 5. Individual frames taken out of an overview movie, where the tip is removed from the test scanning piezotube and transferred to the tip storage/ preparation stage. The field of view is turned by $\sim 90^{\circ}$ relative to that of Fig. 4. Frames (a) and (b) show the approach of the gripper jaws toward the tip ( $y$ and $z$ direction, respectively). (c) Clamping of the tip. (d) Removal of the tip from the scanning piezotube. (e) Transfer of the tip to the tip storage/ preparation stage. (f) Approach of the tip toward the tip storage/preparation site. (g) Insertion of the tip into the tip storage/preparation site. (h) Retraction of the gripper module.

of the tip clamping, removal, and insertion process at the scanning piezotube (Fig. 4). Figure 4 show how the opened gripper jaws approach the tip situated in the scanning piezotube [frames (a) and (b) photographed using a test system in air for better optical access]. Once the gripper module is in the correct position, the two jaws are closed to clamp the tip [compare the jaw separation between frames $(\mathrm{a} / \mathrm{b})$ and $(\mathrm{c})]$. The clamping itself is realized by turning the stepping motor of the gripper module acting of the levers, on which the jaws are mounted. When the tip is tightly clamped the gripper module is vertically lifted without any tilt (d), until the tip is extracted from the scanning piezotube. This process requires the abovementioned perfect alignment between the $z$ axis of the tip manipulator and the scanning piezotube. Once the tip is extracted it can be transferred to any desired position within the reach of the tip manipulator (e). The same process can be inverted as shown in frames (f)-(h). The gripper jaws holding the tip are first approached to the exact vertically aligned position above the scanning tube. Then the whole gripper module including the clamped tip is lowered such that the tip is inserted into the scanning piezotube. Once the final tip position is reached the gripper jaws are opened and retracted to free the space for the sample holder.

Figure 5 shows the transfer of the tip from the scanning piezotube of the abovementioned test STM system into the tip storage/preparation stage. The view is now rotated by roughly $90^{\circ}$ and illustrates the manipulation process from the side of the gripper module. The tip is extracted from the scanning piezotube in a similar manner as shown in Figs. 4(a)-4(d) [Figs. 5(a)-5(c)]. Then the tip is transferred by rotation of the manipulation toward the tip storage stage [Figs. 5(d)-5(f)], where the tip is inserted [Fig. 5(g)] and once done the gripper module is retracted to free the space for potential tip preparation processes.

\section{SUMMARY}

In conclusion, we demonstrated the three-dimensional manipulation of bare STM tips without tip holder by a newly designed three-dimensionally movable manipulator system. The core components of the manipulator system are a spindle guidance for the rotation and $z$ motion driven by two stepping motor-worm gear units. On top of the spindle guidance, a gripper module is mounted, which is moved by piezomotors. The gripper module clamps the bare tips with two hardened tool steel jaws, which are controlled by another stepping motor through two levers, to increase the clamping force, such that the tips cannot slide, shift, or tilt. The extremely stiff and rigid system is directly mounted on the ground plate of the STM and consists of only ultrahigh vacuum compatible components. The system allows the reproducible manipulation of bare tungsten tips made of wires with diameters of $0.25 \mathrm{~nm}$ and having length of only up to 3 $\mathrm{mm}$ without damaging the tip or the STM. The tip manipulators' advantage is the reduction of total mass of the scanning piezotube by removal of a separate tip holder. Thereby, it becomes possible to further increase the resonance frequencies and thus the scanning speed of the STM.

\section{ACKNOWLEDGMENTS}

The authors thank the Deutsche Forschungsgemeinschaft for financial support.

${ }^{1}$ G. Binnig and H. Rohrer, Rev. Mod. Phys. 59, 615 (1987).

${ }^{2}$ R. J. Hamers, R. M. Tromp, and J. E. Demuth, Phys. Rev. Lett. 56, 1972 (1986).

${ }^{3}$ R. M. Feenstra, J. A. Stroscio, and A. P. Fein, Surf. Sci. 181, 295 (1987).

${ }^{4} \mathrm{Ph}$. Ebert, Surf. Sci. Rep. 33, 121 (1999); Curr. Opin. Solid. State Mater. Sci. 5, 211 (2001).

${ }^{5}$ M. B. Johnson, O. Albrektsen, R. M. Feenstra, and H. W. M. Salemink, Appl. Phys. Lett. 63, 2923 (1993); (erratum) 64, 1454 (1994); Ph. Ebert, T. Zhang, F. Kluge, M. Simon, Z. Zhang, and K. Urban, Phys. Rev. Lett. 83, 757 (1999); G. Mahieu, B. Grandidier, D. Deresmes, J. P. Nys, D. Stiévenard, and Ph. Ebert, ibid. 94, 026407 (2005).

${ }^{6}$ Ph. Ebert, C. Domke, and K. Urban, Appl. Phys. Lett. 78, 480 (2001); Ph. Ebert, L. Ivanova, S. Borisova, H. Eisele, A. Laubsch, and M. Dähne, ibid. 94, 062104 (2009).

7 . Albrektsen, D. J. Arent, H. P. Meier, and H. W. M. Salemink, Appl. Phys. Lett. 57, 31 (1990); R. M. Feenstra, Semicond. Sci. Technol. 9, 2157 (1994).

${ }^{8}$ R. M. Feenstra, D. A. Collins, D. Z.-Y. Ting, M. W. Wang, and T. C. McGill, Phys. Rev. Lett. 72, 2749 (1994); J. Steinshnider, J. Harper, M. Weimer, C.-H. Lin, S. S. Pei, and D. H. Chow, ibid. 85, 4562 (2000); W. Barvosa-Carter, M. E. Twigg, M. J. Yang, and L. J. Whitman, Phys. Rev. B 63, 245311 (2001); S. Landrock, K. Urban, and Ph. Ebert, Phys. Rev. 
Lett. 96, 076101 (2006).

${ }^{9}$ N. Liu, J. Tersoff, O. Baklenov, A. L. Holmes, Jr., and C. K. Shih, Phys. Rev. Lett. 84, 334 (2000); B. Grandidier, Y. M. Niquet, B. Legrand, J. P. Nys, C. Priester, D. Stiévenard, J. M. Gérard, and V. Thierry-Mieg, ibid. 85, 1068 (2000); H. Eisele, O. Flebbe, T. Kalka, F. Heinrichsdorff, A. Krost, D. Bimberg, and M. Dähne-Prietsch, Phys. Status Solidi B 215, 865 (1999); H. Eisele, A. Lenz, R. Heitz, R. Timm, M. Dähne, Y. Temko, T. Suzuki, and K. Jacobi, J. Appl. Phys. 104, 124301 (2008).

${ }^{10}$ N. D. Jäger, K. Urban, E. R. Weber, and Ph. Ebert, Phys. Rev. B 65, 235302 (2002); Appl. Phys. Lett. 82, 2700 (2003).

${ }^{11} \mathrm{Ph}$. Ebert and K. Urban Ultramicroscopy 49, 344 (1993); C. Domke, M. Heinrich, Ph. Ebert, and K. Urban, J. Vac. Sci. Technol. B 16, 2825 (1998).

${ }^{12}$ Y. Dong, R. M. Feenstra, R. Hey, and K. H. Ploog, J. Vac. Sci. Technol. B 20, 1677 (2002); S. Landrock, Y. Jiang, K. H. Wu, E. G. Wang, K. Urban, and Ph. Ebert, Appl. Phys. Lett. 95, 072107 (2009); H. Eisele, L. Ivanova, S. Borisova, M. Dähne, M. Winkelnkemper, and Ph. Ebert, ibid. 94, 162110 (2009).
${ }^{13}$ M. Bode, Rep. Prog. Phys. 66, 523 (2003).

${ }^{14}$ S. Heinze, M. Bode, A. Kubetzka, O. Pietzsch, X. Nie, S. Blügel, and R. Wiesendanger, Science 288, 1805 (2000); H. Yang, A. R. Smith, M. Prikhodko, and W. R. L. Lambrecht, Phys. Rev. Lett. 89, 226101 (2002).

${ }^{15}$ L. A. Hockett and S. E. Creager, Rev. Sci. Instrum. 64, 263 (1993); L. Ottaviano, L. Lozzi, and S. Santucci, ibid. 74, 3368 (2003); H. F. Ding, J. E. Pearson, D. Li, R. Cheng, F. Y. Fradin, and S. D. Bader, ibid. 76, 123703 (2005); G. J. de Raad, P. M. Koenraad, and J. H. Wolter, J. Vac. Sci. Technol. B 17, 1946 (1999); T. Kalka, C. Preinesberger, S. Vandré, and M. Dähne-Prietsch, Appl. Phys. A: Mater. Sci. Process. 66, S1073 (1998).

${ }^{16}$ Most commercial STM systems provide tip holders to manipulate the tips within the vacuum system. An improvement of the tip holders provided by Omicron is presented, e.g., in N. Ishida, A. Subagyo, A. Ikeuchi, and K. Sueoka, Rev. Sci. Instrum. 80, 093703 (2009).

${ }^{17}$ L. Kuipers, M. S. Hoogeman, and J. W. M. Frenken, Phys. Rev. Lett. 71, 3517 (1993).

${ }^{18}$ K. Besocke, Surf. Sci. 181, 145 (1987). 